\title{
GENE DISPERSION IN HETEROGENEOUS POPULATIONS
}

\author{
J. G. SKELLAM
}

The Nature Conservancy, London

Received 29.iii.5I

IN a recent article in Heredity, A. J. Bateman (1950) asks "Is gene dispersion normal ?" and assembles a considerable body of evidence to support the general rule that diffusionary processes in populations of living organisms give rise to spatial distributions which are leptokurtic.

In discussing the origin of leptokurtosis he states "There is an apparent contradiction between such a situation and the general theory of normal distributions. Under this theory normality should result whenever a large number of uncorrelated influences are at work. The contradiction forces us to the conclusion that neither the passive nor active movement of organisms is at random."

There are, however, alternative explanations which to my mind seem likely. For example, if we consider the positions taken up by the members of a whole population differing among themselves in their activity or powers of dispersion, we can prove-without abandoning the simplifying assumption of randomness-that a leptokurtic distribution must arise of necessity.

For if the position of a particular organism moving randomly in one dimension has probability function

$$
f(x)=\exp \left\{-\frac{1}{2} x^{2} / \sigma^{2}\right\} / \sigma \sqrt{ }(2 \pi)
$$

and therefore moment generating function

$$
\phi(t)=\exp \left\{\frac{1}{2} \sigma^{2} t^{2}\right\}
$$

and if the value of $\sigma^{2}$ varies between the organisms in any manner whatsoever-say with cumulative distribution function $F\left(\sigma^{2}\right)$-then the probability distribution of the position occupied by an organism chosen at random will have moment generating function

$$
\bar{\phi}(t)=\int_{0}^{\infty} \exp \left\{\frac{1}{2} \sigma^{2} t^{2}\right\} d \mathrm{~F}\left(\sigma^{2}\right)=\mathrm{M}\left(\frac{1}{2} t^{2}\right)
$$

where $\mathbf{M}(\tau)=\Sigma \dot{\mu}_{r} \tau^{r} / r !=\exp \Sigma_{\kappa_{r}} \tau^{r} / r !$

is the moment generating function of the distribution of $\sigma^{2}$.

The cumulant generating function of the required distribution is

$$
\begin{aligned}
\log \bar{\phi}(t) & =\kappa_{1}\left(\frac{1}{2} t^{2}\right)+\frac{1}{2} \kappa_{2}\left(\frac{1}{2} t^{2}\right)^{2}+\ldots \\
& =\kappa_{1} t^{2} / 2 !+3 \kappa_{2} t^{4} / 4 !+\ldots
\end{aligned}
$$

Since $\kappa_{2}>0$ by the hypothesis that $\sigma^{2}$ varies, it follows that the $4^{\text {th }}$ 
cumulant of this distribution is positive and the distribution leptokurtic by definition.

Looking at this result in reverse we see that the second $k$-statistic of the observed distribution is an unbiassed estimate of the mean of $\sigma^{2}$ and that $\frac{1}{3}$ the fourth $k$-statistic of the distribution is an unbiassed estimate of the variance of $\sigma^{2}$. In general

$$
\text { Expectation }\left\{k_{2 n^{2}}{ }^{n} n ! /(2 n) !\right\}=\kappa_{n} \text {. }
$$

Let us now consider the effect of a further period of dispersion on the form of the distribution. For a particular individual the probability distribution will still be normal, and the variance of the final distribution will be the sum of those holding for the two periods separately.

If now the individuals of the population being considered maintain the same relative differences in their powers of dispersal during the second period as during the first, the effect of this extension in time is merely to change the scale of $\sigma^{2}$. The value of $\kappa_{2} / \kappa_{1}{ }^{2}$ therefore remains invariant and as a result of (I) the degree of leptokurtosis is unchanged.

If, however, the activities of the individual organisms vary with time depending say on age or physiological condition, then the relative differences between them on any single day will be greater than those displayed on the average over a longer period. In such circumstances the degree of leptokurtosis must decrease in time (as indeed it has been observed to do), though it will not disappear entirely if the individual differences in activity are not completely smoothed out in the long run.

Theoretical distributions capable of graduating data on this subject may be deduced providing that reasonable assumptions are made regarding the variation in the dispersive powers of the individuals.

As an illustration consider the two dimensional case in which the position of a particular organism has density function

$$
f(x, y)=\exp \left\{-\left(x^{2}+y^{2}\right) / v\right\} / \pi v
$$

where $v=2 \sigma^{2}$ is the mean square deviation from the origin.

The simple and very reasonable assumption that $w \equiv \mathrm{I} / v$ is distributed among individuals as a gamma variate with density function

$$
g(w ; p, \lambda)=e^{-p w} w w^{\lambda-1} p^{\lambda} / \Gamma(\lambda)
$$

leads to the distribution

$$
\begin{aligned}
\bar{f}(x, y) & =\int_{0}^{\infty} \exp \left\{-w\left(x^{2}+y^{2}\right)-p w\right\} w^{\lambda} p^{\lambda} d w / \pi \Gamma(\lambda) \\
& =\frac{\lambda}{\pi p} /\left(\mathrm{I}+\frac{x^{2}+y^{2}}{p}\right)^{\lambda+1}
\end{aligned}
$$

In polar form this distribution is

$$
d P(r)=p^{-1} 2 \lambda r d r /\left(\mathrm{I}+\frac{r^{2}}{p}\right)^{\lambda+1}
$$


The following relations may then be deduced :-

(a) The proportion of the population to be expected outside a circle of radius $R$ is

$$
\int_{R}^{\infty} d \mathrm{P}(r)=\left(\mathrm{I}+\frac{\mathrm{R}^{2}}{p}\right)^{-\lambda}
$$

(b) The mean value of $r$ is $\frac{1}{2} p^{\frac{1}{2}} \pi^{\frac{1}{2}} \Gamma\left(\lambda-\frac{1}{2}\right) / \Gamma(\lambda)$.

(c) The mean value of $r^{2}$ is $p /(\lambda-\mathrm{I})$.

A set of 200 observations on the wanderings of 25 immature millipedes (Blaniulus guttulatus Bosc.) taken from the same nest is shown in the fig. The variate $r$ is the radial distance travelled in

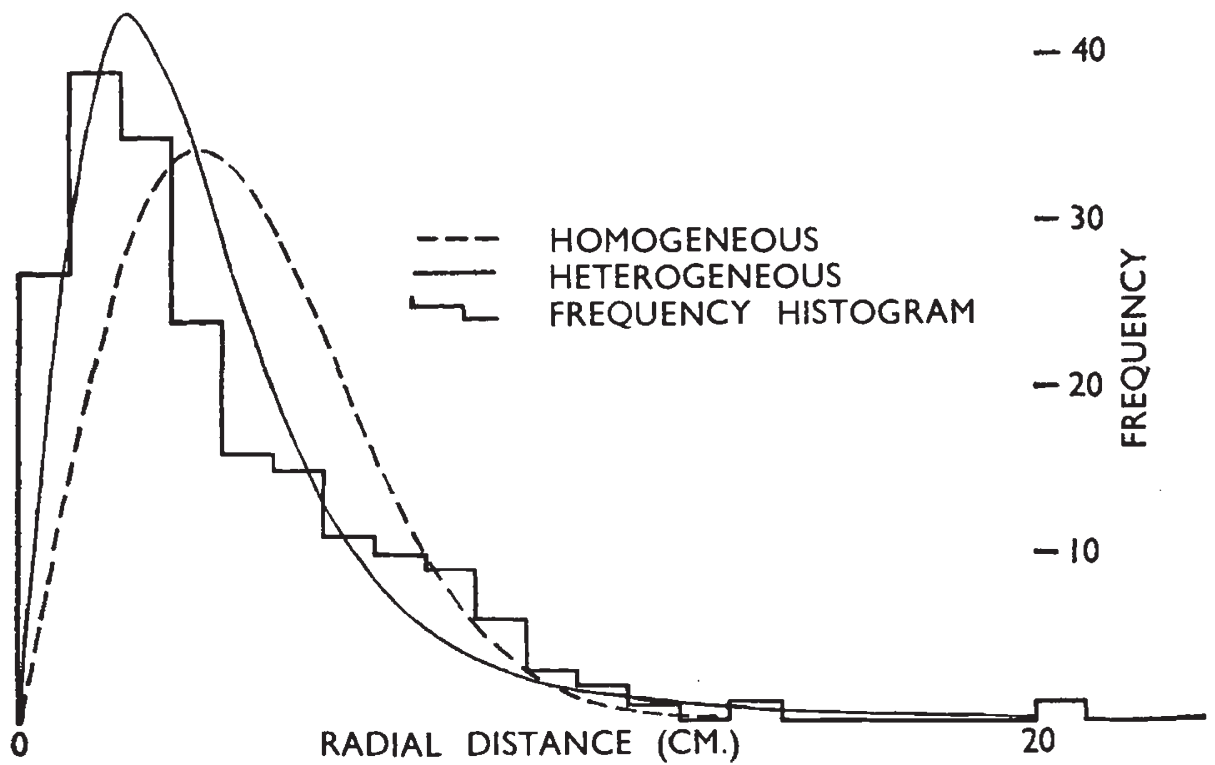

cms. in one minute. Function (2) given above is fitted to the data by the method of moments and compared with the function

$$
f_{o}(r)=e^{-r^{2} / v} 2 r / v
$$

given by normal theory on the assumption of homogeneity. For this function the maximum likelihood estimate of $v$ is simply the mean square dispersion of the observations from the origin.

In the same way, leptokurtic distributions can be deduced for the expected location of offspring if the parent wanders during the period of its reproductive life.

\section{REFERENCES}

BAtEMAN, A. J. 1950. Is gene dispersion normal ? Heredity, 4, 253-263.

SKELLAM, J. G. 1951. Random dispersal in theoretical populations. Biometrika, 38, 196-218. 